\title{
Fuelling of Circumnuclear Regions: 3D Spectroscopy View.
}

\author{
A. V. Moiseev, and A. A. Smirnova \\ Special Astrophysical Observatory, Russian Academy of Sciences, 369167 Russia \\ email:moisav@sao.ru
}

\begin{abstract}
We review the results recently obtained by means of panoramic (3D) spectroscopy on the 6-m telescope BTA (SAO RAS). This research is devoted to observational evidences of gas inflow into the centers of galaxies, which must ensure the fueling of an active nucleus. 3D spectroscopy allows us to trace the behavior of gas and stars in the one-kiloparsec size vicinity of an AGN and also far from the center.
\end{abstract}

Keywords. galaxies: Seyfert, galaxies: jets, galaxies: kinematics and dynamics

\section{NGC 6104 (Sy1.5)}

We analyzed the structure of NGC6104 on various spatial scales (Smirnova et al., 2006a): (i) At $r<100-200 \mathrm{pc}$ we found effects explained in terms of the interaction between a radio jet and an ambient interstellar medium. (ii) At $r=1-5 \mathrm{kpc}$, we identified radial motions of ionized gas along the bar towards the nucleus with the velocities of $50 \pm 10 \mathrm{~km} / \mathrm{s}$. We measured the bar pattern speed and showed an absence of the ILR. Therefore, the radial gas motions can reach the nucleus and fueling its central engine. (iii) At $r=25-45 \mathrm{kpc}$, we detected extended asymmetric filaments of low surface brightness. This structure resembles most closely a companion disrupted by tidal forces.

\section{Mrk 533 (Sy 2)}

Amram et al. (2003) have published the $\mathrm{H} \alpha$ velocity field of this main member of the compact group HCG96 and mentioned an asymmetry in the different sides of its rotation curve. We present a careful analysis of the velocity fields in the different emission lines and found numerous peculiarities in the gas motion (Smirnova et al., 2006b): (i) The streaming motions along the bar were found from a 'tilted-ring' velocity analysis.

(ii) The map of radial motions in the disc shows the gas inflow along the Northern arm $\left(5^{\prime \prime}<r<15^{\prime \prime}\right)$. (iii) The circumnuclear outflow in $r<5^{\prime \prime}$ along galactic minor axis was also detected. We assume that these ionized gas outflows are triggered by the radio jet intrusion in an ambient medium.

\section{Acknowledgements}

This work was supported by the RFBR grant 06-02-16825.

\section{References}

Amram, P., Plana, H., Mendes de Oliveira, C., Balkowski, C., \& Boulesteix, J., 2003, A $\& A$, 402, 865.

Smirnova, A.A., Moiseev, A.V., \& Afanasiev, V.L., 2006a, Astronomy Letters, 32, 520, astro$\mathrm{ph} / 0607163$.

Smirnova A.A., Gavrilović, N., Moiseev, A.V., \& Popović, L., Č. et al., 2006b, MNRAS, submitted. 